\title{
Impactos da ventilação mecânica invasiva em pacientes de COVID-19: revisão
}

\section{integrativa}

\author{
Impacts of invasive mechanical ventilation on patients from COVID-19: integrative review \\ Impactos de la ventilación mecánica invasiva en pacientes de COVID-19: revisión integrativa
}

Recebido: 20/08/2021 | Revisado: 25/08/2021 | Aceito: 01/09/2021 | Publicado: 04/09/2021

\author{
Daniel Alves Cruz \\ ORCID: https://orcid.org/0000-0002-3585-2794 \\ Universidade Federal do Piauí, Brasil \\ E-mail: daniel3404558@gmail.com \\ Ismaília de Lima Sousa \\ ORCID: https://orcid.org/0000-0002-5027-7652 \\ Universidade Federal do Piauí, Brasil \\ E-mail: ismhailliamartins10@outlook.com \\ Paloma Veluma Dias Santana \\ ORCID: https://orcid.org/0000-0003-2454-7740 \\ Universidade Federal do Piauí, Brasil \\ E-mail: paloma_veluma@hotmail.com \\ Luciana Karine De Abreu Oliveira \\ ORCID: https://orcid.org/0000-0002-9314-0234 \\ Universidade Federal do Piauí, Brasil \\ E-mail: alucianakarine@ outlook.com \\ Francisco Wagner dos Santos Sousa \\ ORCID: https://orcid.org/0000-0001-9309-2925 \\ Universidade Estadual do Piauí, Brasil \\ E-mail: wagnersantosreal@gmail.com \\ Ágata Maria Xavier de Araújo \\ ORCID: https://orcid.org/0000-0001-8442-4748 \\ Universidade Estadual do Piauí, Brasil \\ E-mail: agatamaryibs@hotmail.com \\ Keila Maria Paiva da Silva \\ ORCID: https://orcid.org/0000-0003-0928-484X \\ Universidade Federal do Piauí, Brasil \\ E-mail: keilapaiva2014@gmail.com \\ Glória Stéphany Silva de Araújo \\ ORCID: https://orcid.org/0000-0002-6473-2724 \\ Universidade Estadual do Piauí, Brasil \\ E-mail: gloriastephany360@gmail.com \\ Jayanne do Nascimento Santana Costa \\ ORCID: https://orcid.org/0000-0003-1034-0028 \\ Universidade Estadual do Piauí, Brasil \\ E-mail: jayns9antana@outlook.com \\ Isabel Rodrigues do Nascimento \\ ORCID: https://orcid.org/0000-0002-3744-6968 \\ Universidade Federal do Piauí, Brasil \\ E-mail: nascimentoisabe1620@gmail.com
}

\begin{abstract}
Resumo
A utilização da ventilação mecânica invasiva (VMI) tornou-se uma importante alternativa para os pacientes criticamente enfermos pela COVID-19, pois essa terapêutica disponibiliza aos assistidos em internações hospitalares o oxigênio necessário, diminuindo a mortalidade nas unidades assistenciais. Dessa forma, o presente estudo objetivou descrever os impactos da ventilação mecânica invasiva em pacientes vítimas de COVID-19. As pesquisas foram feitas nos periódicos eletrônicos Lilacs, PubMed, Scopus e MedLine. Utilizando a questão norteadora: Quais as repercussões da ventilação mecânica invasiva no tratamento de pacientes com COVID-19? Como resultados, indicou que há muitas vezes aumento nas chances de óbitos nos acometidos, bem como, o mau uso da VMI também pode complicar o tratamento terapêutico preconizado. Portanto, os impactos comuns em pacientes vítimas de COVID-19 em ventilação mecânica invasiva resultam em lesão pulmonar ou agravamento das condições clínicas do paciente, como aparecimento de barotrauma, atelectasia, causando pressão insuficiente durante o suporte ventilatório.
\end{abstract}

Palavras-chave: Ventilação mecânica; Coronavírus; Respiração artificial; COVID-19. 


\begin{abstract}
The use of invasive mechanical ventilation (IMV) has become an important alternative for critically ill patients due to COVID-19, as this therapy provides those assisted in hospital admissions with the necessary oxygen, reducing mortality in healthcare units. Thus, the present study aimed to describe the impacts of invasive mechanical ventilation on patients victims of COVID-19. Searches were carried out in the electronic journals Lilacs, PubMed, Scopus and MedLine. Using the guiding question: What are the repercussions of invasive mechanical ventilation in the treatment of patients with COVID-19? As a result, it indicated that there is often an increase in the chances of death in those affected, as well as the misuse of IMV can also complicate the recommended therapeutic treatment. Therefore, the common impacts on patients victims of COVID-19 under invasive mechanical ventilation result in lung injury or worsening of the patient's clinical conditions, such as the appearance of barotrauma, atelectasis, causing insufficient pressure during ventilatory support.
\end{abstract}

Keywords: Mechanical ventilation; Coronavirus; Artificial Respiration; COVID-19.

\title{
Resumen
}

El uso de ventilación mecánica invasiva (VMI) se ha convertido en una alternativa importante para los pacientes críticos debido al COVID-19, ya que esta terapia proporciona a los asistidos en ingresos hospitalarios el oxígeno necesario, reduciendo la mortalidad en las unidades de salud. Por lo tanto, el presente estudio tuvo como objetivo describir los impactos de la ventilación mecánica invasiva en pacientes víctimas de COVID-19. Las búsquedas se realizaron en las revistas electrónicas Lilacs, PubMed, Scopus y MedLine. Utilizando la pregunta orientadora: ¿Cuáles son las repercusiones de la ventilación mecánica invasiva en el tratamiento de pacientes con COVID-19? En consecuencia, indicó que a menudo hay un aumento de las posibilidades de muerte en los afectados, así como el mal uso de la VMI también puede complicar el tratamiento terapéutico recomendado. Por lo tanto, los impactos comunes en pacientes víctimas de COVID-19 bajo ventilación mecánica invasiva resultan en lesión pulmonar o empeoramiento de las condiciones clínicas del paciente, como la aparición de barotrauma, atelectasia, causando presión insuficiente durante el soporte ventilatorio.

Palabras clave: Ventilación mecánica; Coronavirus; Respiración artificial; COVID-19.

\section{Introdução}

A pandemia do SARS-CoV-2, o novo coronavírus que é responsável pela doença COVID-19, apresentou os primeiros casos em dezembro de 2019, em Wuhan, na China (World Health Organization, 2020). A partir disso, tornou-se um grande desafio para todos os sistemas de saúde do mundo, caracterizado como um grave problema de saúde pública e sem antecedentes, com elevadas taxas de internação e de mortalidade. Nessa perspectiva, o COVID-19 é uma doença aguda em que o seu quadro pode ser revertido, mas que, também pode ser mortal principalmente quando afeta grupos de risco, como idosos e pessoas diagnosticadas com doenças crônicas e/ou prévias (Campos \& Costa, 2020; Rodrigues et al., 2016).

A ventilação mecânica invasiva (VMI) tornou-se uma imprescindível modalidade terapêutica para pessoas que desenvolveram complicações de COVID-19 em terapia intensiva. Por meio dela, é possível salvar vidas de pacientes que apresentam dificuldade respiratória grave, uma vez que garante repouso da musculatura respiratória e uma oxigenação apropriada. Todavia, o índice de mortalidade de pacientes acometidos por COVID-19 que necessitam de VMI ainda é alarmante, quando se trata de pacientes longevos, embora estejam assistidos por um sistema de saúde adequado (King et al., 2020; Prazeres et al., 2021; Silva et al., 2017).

No atual cenário pandêmico, os hospitais passaram a enfrentar diversos desafios, dentre eles, estruturais e assistenciais. Tal fato é perceptível pelo déficit de leitos de UTI aparelhado com ventiladores mecânicos, e também por indagações pertinentes à infraestrutura, treinamento das equipes de saúde, manutenção de equipamentos e recursos humanos. Dessarte, isso contribuiu para que houvesse a reutilização de equipamentos de uso único e precária assistência ao usuário. É válido salientar, ainda, que para atuar na UTI trabalhando com o suporte ventilatório complexo fazem-se necessários um treinamento eficaz e de qualidade para a equipe de saúde, a fim de mitigar outras intercorrências imprevisíveis (Dondorp, Hayat, Aryal, Beane \& Schultz, 2020). 
A VMI gera lesões biofísicas e bioquímicas, podendo ocasionar agravos pulmonares e morte. Assim, é essencial que os cuidados para com o paciente em suporte ventilatório sejam bem reconhecidos pelos profissionais da saúde, a fim de impedir o desenvolvimento de uma lesão relacionada à ventilação mecânica.

Diante disso, este estudo teve como objetivo descrever os impactos da ventilação mecânica invasiva em pacientes de COVID-19, a partir de busca na literatura de estudos publicados entre os anos de 2019 à 2021, na tentativa de desenvolver a excelência na assistência à saúde.

\section{Metodologia}

Trata-se de uma de revisão integrativa da literatura (RIL), realizada no período de abril a agosto de 2021. A pergunta norteadora da pesquisa foi construída com o auxílio da estratégia PICO: Problema /fenômeno (P) = COVID-19; Intervenção /interesse (I) = Utilização da ventilação mecânica invasiva; Contexto/característica (Co) = Serviços de saúde. Assim, o delineamento deste estudo surgiu a partir da seguinte questão de pesquisa: Quais as repercussões da ventilação mecânica invasiva no tratamento de pacientes com COVID-19?

Para a realização desta pesquisa, foram realizadas cinco etapas, a saber: formulação de questões de pesquisa clara e objetiva com base na determinação do problema; busca e amostragem de estudos primários na literatura científica, de acordo com os padrões de inclusão e exclusão previamente determinados para a pesquisa; análise e caracterização rigorosa da pesquisa que compõe o estudo; síntese do conhecimento e apresentação da revisão (Mendes, Pereira \& Galvão, 2019; Souza, Silva \& Carvalho, 2010).

As bases de dados utilizadas para a busca foram: LILACS, PubMed, Scopus e MedLine. Foram utilizados os seguintes descritores em Ciências da Saúde (DeCS) e descritores booleanos na seguinte combinação: (Ventilação mecânica) OR (Respiração artificial) AND (Coronavírus) OR (COVID-19). Os descritores foram inseridos na língua portuguesa e em suas respectivas versões para os idiomas: inglês e espanhol.

Os critérios de inclusão compreenderam: artigos originais publicados na íntegra, disponíveis online nas bases de dados, nos idiomas português, inglês e espanhol, em um recorte temporal entre os anos 2019 a 2021 (até o mês de julho), esse recorte servirá para caracterizar melhor a temática estudada, na tentativa de sintetizar o conhecimento e as experiências nesse período de tempo. Serão excluídos os artigos repetidos, que não respondam à questão de pesquisa, artigos de revisão da literatura, teses, dissertações, livros, editoriais, resumos de eventos e relatos de casos ou artigos sem qualquer relação com os objetivos da pesquisa, por meio da leitura de título e resumo.

A estratégia de busca em bases de dados por cruzamento dos descritores resultou em um total de 796 publicações, que foram exportados para o software Rayyan ${ }^{\circledR}$ para a seleção da amostra, sendo 10 oriundas da Lilacs, 202 vindos da Scopus, 410 da MedLine e 174 da PubMed. Inicialmente com a utilização do software foram identificados e excluídos os estudos duplicados $(\mathrm{n}=459)$. Após a exclusão dos duplicados, os pesquisadores procederam a leitura dos títulos e resumos dos 337 estudos restantes, de forma independente, fazendo seus julgamentos e aplicando os critérios de inclusão e exclusão individualmente.

No segundo momento, os dois revisores, a partir do consenso, indicaram os artigos pré-selecionados, definidos trinta e quatro artigos que se adequaram nos critérios de elegibilidade para a leitura do texto na íntegra. No terceiro momento, os dois revisores leram os artigos de forma independente $(\mathrm{n}=34)$ e, com o auxílio do terceiro revisor, foram determinadas as qualificações finais, dentre elas, apenas doze artigos constituíram a amostra final da revisão.

Seguindo as recomendações do Preferred Reporting Items for Systematic Reviews and MetaAnalyses -PRISMA® , a revisão foi produzida de forma sistematizada ( Sousa, Firmino, Marques-Vieira, Severino \& Pestana., 2018; Moher, Liberati, 
Tetzlaff, Altman \& Prisma Group, 2009). Por trata-se de uma revisão, conforme resolução CNS nº 466/12, o presente estudo não foi submetido à apreciação do Comitê de Ética em Pesquisa em Seres Humanos. O processo completo de seleção da amostra dos artigos pode ser visto no fluxograma da Figura 1.

Figura 1 - Fluxograma PRISMA de seleção da amostra dos artigos. Teresina (PI), Brasil, 2021.

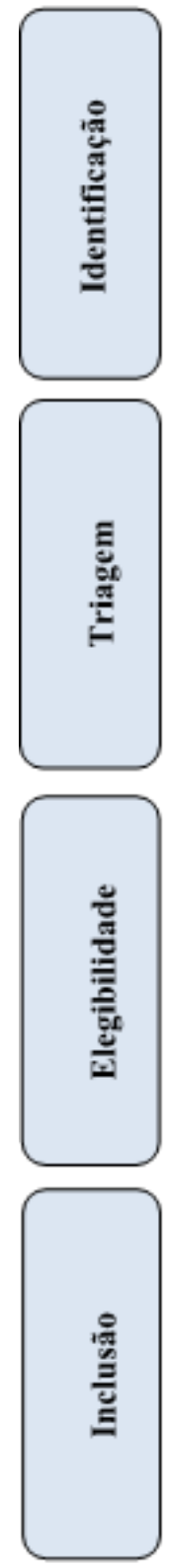

Estudos identificados nas bases de dados:

LILACS $(n=10)$, MedLine $(n=410)$, Scopus $(n=202)$, PubMed $(n=174)$

Total $(\mathrm{n}=796)$

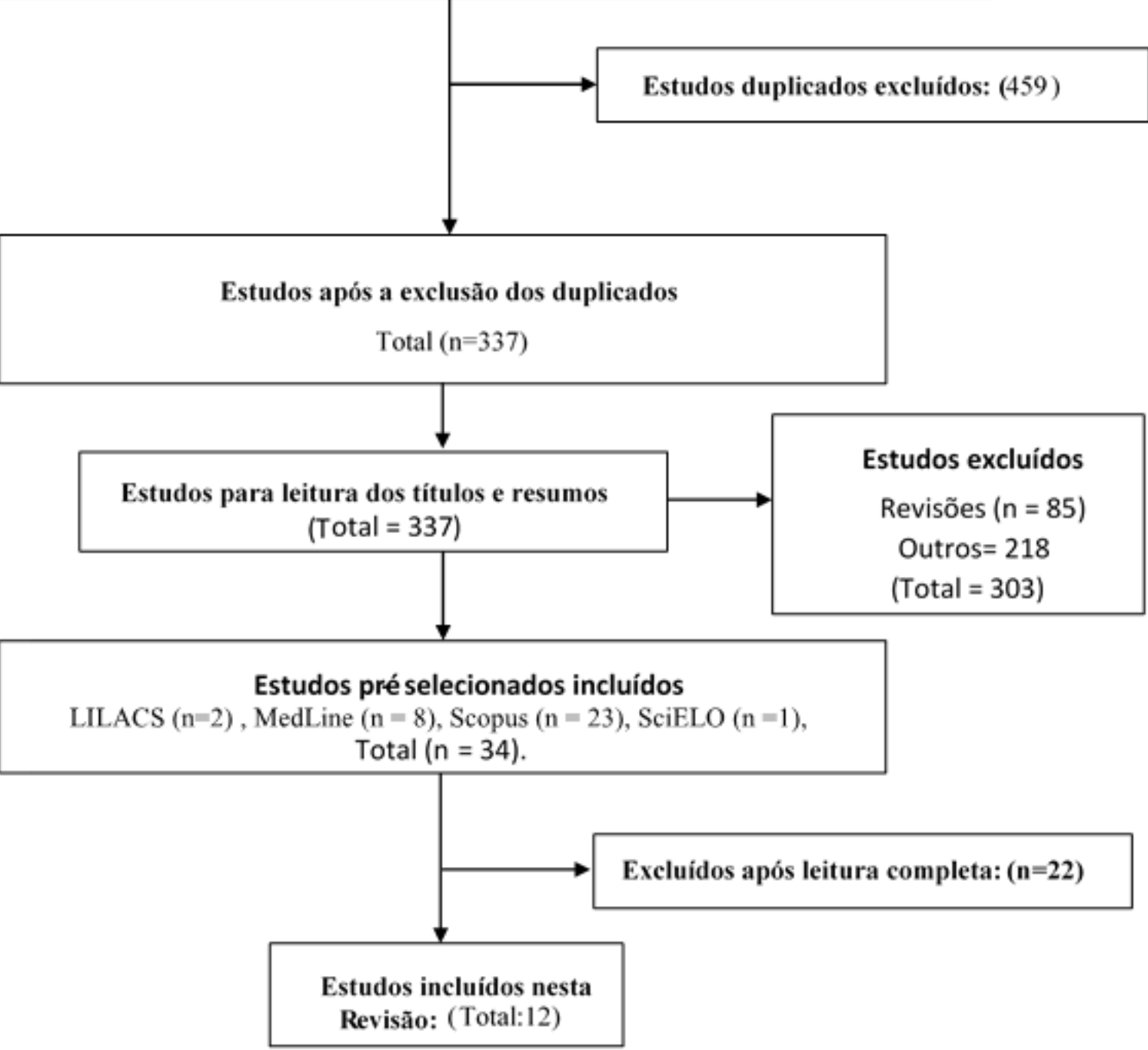

Fonte: Autores (2021)

\section{Resultados e Discussão}

Na presente pesquisa, um total de doze estudos foram escolhidos por responder a questão norteadora e os objetivos anteriormente escolhidos. Os seguintes dados foram extraídos a partir dos artigos: referências, periódico, base de dados, 
objetivo do estudo e metodologia. Em relação à base de dados, sete foram encontradas na PubMed (58\%), duas na Scopus $(16,8 \%)$, dois na MedLine (16,8\%), e um na LiLacs $(8,4 \%)$.

Quadro 1. Caracterização dos estudos.

\begin{tabular}{|c|c|c|c|c|}
\hline Referências & Periódico & $\begin{array}{c}\text { Base de } \\
\text { dados }\end{array}$ & Objetivos & Metodologia \\
\hline $\begin{array}{l}\text { Dondorp, Hayat, } \\
\text { Aryal, Beane \& } \\
\text { Schultz, } 2020\end{array}$ & $\begin{array}{l}\text { The American journal } \\
\text { of tropical medicine } \\
\text { and hygiene }\end{array}$ & PubMed & $\begin{array}{l}\text { Discutir os principais aspectos no gerenciamento da } \\
\text { COVID-19: ventilação apoio, com um foco na restrição e } \\
\text { limitação desses recursos. }\end{array}$ & $\begin{array}{l}\text { Estudo } \\
\text { retrospectivo, } \\
\text { observacional }\end{array}$ \\
\hline Protti et al., 2020 & $\begin{array}{c}\text { Minerva } \\
\text { anestesiologica }\end{array}$ & PubMed & $\begin{array}{c}\text { Descrever a incidência e os fatores de risco para } \\
\text { barotrauma em pacientes com o novo coronavírus } \\
\text { (COVID-19) submetidos à ventilação mecânica invasiva } \\
\text { durante o surto na região de Lombardia, Itália. }\end{array}$ & $\begin{array}{c}\text { Estudo } \\
\text { observacional }\end{array}$ \\
\hline $\begin{array}{l}\text { McGuinness et } \\
\text { al., } 2020\end{array}$ & Radiology & PubMed & $\begin{array}{l}\text { Determinar a taxa de barotrauma em pacientes que } \\
\text { testaram positivo para SARS-CoV-2 e necessitaram de } \\
\text { VMI em comparação a outros pacientes. }\end{array}$ & $\begin{array}{c}\text { Estudo } \\
\text { retrospectivo }\end{array}$ \\
\hline King et al., 2020 & Heart Rhythm & Scopus & $\begin{array}{l}\text { Determinar as características clínicas e os resultados de } \\
\text { pacientes com COVID-19 tratados com ventilação } \\
\text { mecânica invasiva em um sistema de saúde dos Estados } \\
\text { Unidos da América com recursos adequados. }\end{array}$ & $\begin{array}{c}\text { Estudo } \\
\text { observacional }\end{array}$ \\
\hline Li et al., 2020 & $\begin{array}{l}\text { Therapeutic advances } \\
\text { in respiratory disease }\end{array}$ & PubMed & $\begin{array}{l}\text { Identificação os fatores de risco potenciais para ventilação } \\
\text { mecânica invasiva e não invasiva em pacientes com a } \\
\text { doença coronavírus (COVID-19). }\end{array}$ & $\begin{array}{l}\text { Estudo } \\
\text { retrospectivo }\end{array}$ \\
\hline $\begin{array}{c}\text { Cavayas et al., } \\
2021\end{array}$ & $\begin{array}{l}\text { Canadian journal of } \\
\text { anaesthesia }\end{array}$ & PubMed & $\begin{array}{l}\text { Descrever as características tratamentos e resultados de } \\
\text { pacientes com COVID-19 gravemente enfermos em } \\
\text { Montreal. }\end{array}$ & $\begin{array}{l}\text { Estudo de coorte } \\
\text { retrospectivo }\end{array}$ \\
\hline Udi et al., 2021 & $\begin{array}{l}\text { Journal of Intensive } \\
\text { Care Medicine }\end{array}$ & PubMed & $\begin{array}{c}\text { Investigar os casos de barotrauma em pacientes com } \\
\text { pneumonia por COVID-19 que requerem VMI } \\
\text { prolongada. }\end{array}$ & $\begin{array}{l}\text { Estudo de caso } \\
\text { controle }\end{array}$ \\
\hline Zhang et al., 2020 & $\begin{array}{l}\text { Emerging microbes } \\
\quad \& \text { infections }\end{array}$ & MedLine & $\begin{array}{l}\text { Descrever os riscos de infecção secundária, tempo de } \\
\text { ocorrência e taxa de incidência de infecção } \\
\text { relacionada à operação clínica com VMI. }\end{array}$ & $\begin{array}{l}\text { Estudo de coorte } \\
\text { retrospectivo } \\
\text { multicêntrico }\end{array}$ \\
\hline Wang et al., 2020 & Critical care medicine & Scopus & $\begin{array}{c}\text { Delinear as características clínicas de pacientes com } \\
\text { a doença COVID-19 usando ventilação mecânica } \\
\text { invasaiva, por meio de estudo tipo coorte nacional na } \\
\text { China. }\end{array}$ & $\begin{array}{c}\text { Estudo prospectivo } \\
\text { observacional }\end{array}$ \\
\hline $\begin{array}{l}\text { Gamberini et al., } \\
2020\end{array}$ & $\begin{array}{c}\text { Journal of intensive } \\
\text { care }\end{array}$ & Pubmed & $\begin{array}{c}\text { Investigar os fatores de riscos associados com } \\
\text { incapacidades por COVID-19 em pacientes de } \\
\text { ventilação mecânica invasiva, devido a progressão e } \\
\text { complicações durante a ventilação. }\end{array}$ & $\begin{array}{l}\text { Estudo } \\
\text { observacional } \\
\text { prospectivo } \\
\text { multicêntrico }\end{array}$ \\
\hline $\begin{array}{c}\text { Garcia et al., } \\
2021\end{array}$ & PloS one & MedLine & $\begin{array}{c}\text { Desenvolver um score que estima o risco para o uso } \\
\text { da ventilação mecânica invasiva (VMI) em pacientes } \\
\text { com COVID-19. }\end{array}$ & $\begin{array}{l}\text { Estudo de coorte } \\
\text { retrospectivo }\end{array}$ \\
\hline
\end{tabular}




\begin{tabular}{|c|c|c|c|c|}
\hline $\begin{array}{l}\text { Humeres et al., } \\
2020\end{array}$ & $\begin{array}{l}\text { International journal } \\
\text { of odontostomatology }\end{array}$ & Lilacs & $\begin{array}{c}\text { Apresentar um protocolo de manutenção e higiene } \\
\text { bucal para pacientes submetidos à ventilação } \\
\text { mecânica invasiva (VMI) internados em unidades de } \\
\text { terapia intensiva. }\end{array}$ & Estudo prospectivo \\
\hline
\end{tabular}

Fonte: Autores (2021).

Quando se trata dos impactos da ventilação mecânica invasiva em pacientes de COVID-19, observa-se que o mau uso da VMI pode desencadear lesão pulmonar ou acentuar o quadro clínico do paciente, como desenvolver biotrauma (lesão local devido à ação de um agente externo vulnerante), oxitrauma (por radicais livres de oxigênio), atelectrauma, volutrauma e barotrauma como consequência de fluxos, administração de grandes volumes e pressões inadequadas durante o suporte ventilatório (Dondorp, Hayat, Aryal, Beane \& Schultz, 2020). É válido ressaltar a presença de uma diferença significativa nos pulmões que são afetados por COVID-19, em que consiste na coexistência de áreas pulmonares severamente afetadas, adjuntas a áreas relativamente não acometidas.

O estudo desenvolvido em 38 hospitais na Lombardia, Itália, mostrou que houve aumento da incidência e fatores de risco por barotrauma em pacientes com a doença coronavrus (COVID-19) em ventilação mecânica invasiva, durante o surto da pandemia. Os pacientes com COVID-19 aprestavam maiores chances de apresentar o barotrauma durante o uso do suporte ventilatório mesmo sendo supostamente protetora para as lesões pulmonares do que os doentes sem COVID-19 (Protti et al., 2020). Corroborando com isso, a pesquisa realizada com 601 pacientes infectados pelo novo coronavírus que receberam a VMI (71\% do sexo masculino), mostrou que um ou mais eventos de barotrauma ocorreram em 89 destes (15\%) dos pacientes, para um total de 145 casos (24\% do total sem correlação com a doença COVID-19) (McGuinness et al., 2020).

Outro estudo realizado por meio de revisão de prontuário eletrônico, de 1.023 pacientes positivos para COVID-19 admitidos em sistema de saúde referência dos EUA, observou que 164 (16,0\%) destes foram tratados com ventilação mecânico invasiva sendo todos acompanhados até a disposição definitiva do caso. Onde 70/164 (42,7\%) dos pacientes morreu, enquanto 94/164 (57,3\%) estavam ainda vivos. Os pacientes que morreram eram mais velhos em comparação aos demais estudados (idade mediana acima de 66), corroborando que a idade avançada torna-se um importante agravante para a mortalidade (King et al., 2020).

Já o enfoque principal realizado com um total de 516 pacientes com COVID-19 confirmado em laboratório incluídos no estudo de coorte retrospectivo, foi demonstrar, a maior necessidade de utilização de terapêuticas medicamentosas durante a VMI, tais como o interferon alfa, lopinavir / litonavir, ribavirina, antibióticos e glicocorticoide durante o tratamento, comparado com os que não utilizaram VMI. Em adição a isso, a hipóxia, microtrombos e drogas desempenharam um papel importante com danos no fígado provocando agravamento dos assistidos (Li et al., 2020).

A realização da ventilação não invasiva com pressão positiva tevê uma alta taxa de falha (70\%) quando usada em pacientes criticamente enfermos com limitações de tratamento, mostrando a importância da VMI (Cavayas et al., 2021). Além disso, a presença de complicação a exemplo do barotrauma em COVID-19 induzida por insuficiência respiratória em uso da ventilação mecânico invasiva foi encontrado em $40 \%$ dos pacientes incluídos no presente estudo (Udi et al., 2021). Os achados sugerem que o barotrauma em pacientes vítimas da COVID-19 pode ocorrer mesmo se as recomendações para proteção pulmonar VMI forem seguidas.

Ademais, os pacientes que receberam a VMI estavam em estado crítico tiveram maior chance de infecção secundária. As infecções mais comuns identificadas através de coleta de amostras das vias respiratórias, urinária, cateteres, e sangue de acordo com a clínica apresentada foram: infecções respiratórias, do sangue e do sistema urinário. Entre as infecções respiratórias, os patógenos mais detectados consistiram de bactérias gram-negativas, seguidas por bactérias gram-positivas, vírus, fungos e outros (Zhang et al., 2020). Já, os fatores de risco potenciais para níveis elevados de dímero D podem ajudar os 
profissionais da saúde a determinar se a ventilação mecânica invasiva é necessária em um estágio inicial da doença diminuindo o agravamento do quadro dos acometidos (Wang et al., 2020).

Em uma pesquisa realizado utilizando somente suporte ventilatório invasivo em uso do tubo endotraquel ou traqueostomia, 216 pacientes $(55,2 \%)$ tiveram pelo menos uma complicação não pulmonar, sendo o mais comum o aparecimento da lesão renal com a necessidade de terapia renal substitutiva (76 pacientes, 19,4\%) e complicações cardiovasculares (66 pacientes, 16,9\%). Já as complicações pulmonares, a mais comumente observada foi a pneumonia associada à ventilação mecânica invasiva de início tardio (175 pacientes, 44,8\%) (Gamberini et al., 2020).

Portanto, intervenções como a umidificação passiva (filtro HME); segurança com o circuito fechado para aspiração de via aérea; uso de filtro de barreira na válvula exalatória (HEPA); inaladores pressurizados dosimetrados com adaptador para administração de broncodilatadores; 30min/ 1 hora, recolher gasometria, medição do índice de oxigenação e radiografia de tórax após intubação orotraqueal; pressão do balonete do tubo traqueal entre $25-32 \mathrm{cmH}_{2} \mathrm{O}$, checar e evitar vazamentos, sendo tais práticas importantes para a redução de iatrogênias e o risco de mortes dos pacientes (Garcia et al., 2021).

Os pacientes com VMI apresentam uma diminuição significativa na capacidade ventilatória autonômica ao longo do tempo. Além disso, ocorre a perda da deglutição e o reflexo de tosse devido a sedação e impedimento físico, que determina o acúmulo das secreções produzidas pela via respiratória, que fluem para o estabelecimento do local ideal para a entrada e desenvolvimento de microrganismos oportunistas. Além do exposto, a descamação mucosa oral, traumatismo dos dentes, edema grave de língua, úlceras traumáticas e de pressão, boca seca e mucosas secas podem ser fazer presentes possibilitando piora do estado clínico do paciente, caso medidas preventivas e curativas não sejam realizadas (Humeres et al., 2020).

O principal objetivo das intervenções assistenciais com ventilação mecânica invasiva busca nos pacientes gravemente acometidos por COVID-19 hes oferecer e permitir o retorno das funções fisiológicas. Assim, a conduta no manejo dos infectados pela doença deve ser individualizada e baseada no conhecimento da eficácia das medidas utilizadas.

\section{Conclusão}

As alterações fisiopatológicas dos pacientes com COVID-19 que requerem ventilação mecânica invasiva são caracterizadas por mudanças pulmonares abrangentes, como a atelectasia pulmonar. Além disso, foi observado que a VMI quando mau utilizada torna-se um indicador de maior probabilidade de mortalidade, que pode eventualmente promover um ciclo vicioso entre os pulmões e a ventilação mecânica invasiva, aumentado a dependência do paciente com esse tratamento.

Ademais, a taxa de mortalidade dos pacientes com COVID-19 que necessitam da ventilação é alta, sendo observado particularmente naqueles mais idosos cujos índices tendem a piorar em comparação com o restante da população, mesmo em sistemas de saúde com bons recursos. Portanto, grande parte dos pacientes que necessitam de ventilação mecânica invasiva, são idosos e apresentam comorbidades, diminuindo as chances de recuperação e maior mortalidade pela doença COVID-19.

Além disso, dada a relevância do tema, espera-se que outros estudos sejam realizados para abordar a questão da inserção dessa temática durante a graduação, a fim de preparar futuros profissionais para o atendimento humanizado e a implementação de ações de educação em saúde, ampliando os espaços de discussão para além das salas de aula e divulgar o conhecimento sobre a ventilação mecânica invasiva ajudando a combater as consequências evitáveis e prejudiciais à saúde dos pacientes. 
Research, Society and Development, v. 10, n. 11, e380101119656, 2021

(CC BY 4.0) | ISSN 2525-3409 | DOI: http://dx.doi.org/10.33448/rsd-v10i11.19656

\section{Referências}

Campos, N. G. \& Costa, R. F. (2020). Alterações pulmonares causadas pelo novo Coronavírus (COVID-19) e o uso da ventilação mecânica invasiva. J Health Biol Sci. 8(1), 1-3. https://doi: 10.12662/2317-3076jhbs.v8i1.3185.

Cavayas, Y.A., Noël, A., Brunette, V. et al. (2021). Early experience with critically ill patients with COVID-19 in Montreal. Can J Anesth/J Can Anesth 68, 204-213. https://doi.org/10.1007/s12630-020-01816-Z.

Dondorp, A. M., Hayat, M., Aryal, D., Beane, A., \& Schultz, M. J. (2020). Respiratory Support in COVID-19 Patients, with a Focus on Resource-Limited Settings. The American journal of tropical medicine and hygiene, 102(6), 1191-1197. https://doi.org/10.4269/ajtmh.20-0283.

Gamberini, L., Tonetti, T., Spadaro, S., Zani, G., Mazzoli, C. A., Capozzi, C., Giampalma, E., Bacchi Reggiani, M. L., Bertellini, E., Castelli, A., Cavalli, I., Colombo, D., Crimaldi, F., Damiani, F., Fogagnolo, A., Fusari, M., Gamberini, E., Gordini, G., Laici, C., Lanza, M. C., ICU-RER COVID-19 Collaboration (2020). Factors influencing liberation from mechanical ventilation in coronavirus disease 2019: multicenter observational study in fifteen Italian ICUs. Journal of intensive care, 8, 80. https://doi.org/10.1186/s40560-020-00499-4.

Garcia, G. J. A., Camiro-Zúñiga, A., Aguilar-Soto, M., Cuenca, D., Cadena-Fernández, A., Khouri, L. S., Rayek, J. N., Mercado, M., \& ARMII Study Group (2021). COVID-IRS: A novel predictive score for risk of invasive mechanical ventilation in patients with COVID-19. PloS one, 16(4), e0248357. https://doi.org/10.1371/journal.pone.0248357.

Humeres, S. Carlos, Márquez, Agustín, González-Ritchie, Paulina, Valenzuela-Ramos, Ricardo, Rivera-Saavedra, Marcelo, Vásquez, Pablo, \& Araya-Salas, Cristóbal. (2020). Manejo Multidisciplinario de la Cavidad Oral en Pacientes COVID-19 bajo Ventilación Mecánica Invasiva. Rol del Equipo Odontológico. International journal of odontostomatology, 14(4), 701-704. https://dx.doi.org/10.4067/S0718-381X2020000400701.

King, C. S., Sahjwani, D., Brown, A. W., Feroz, S., Cameron, P., Osborn, E., Desai, M., Djurkovic, S., Kasarabada, A., Hinerman, R., Lantry, J., Shlobin, O. A., Ahmad, K., Khangoora, V., Aryal, S., Collins, A. C., Speir, A., \& Nathan, S. (2020). Outcomes of mechanically ventilated patients with COVID-19 associated respiratory failure. PloS one, 15(11), e0242651. https://doi.org/10.1371/journal.pone.0242651.

Li, W., Lin, F., Dai, M., Chen, L., Han, D., Cui, Y., \& Pan, P. (2020). Early predictors for mechanical ventilation in COVID-19 patients. Therapeutic advances in respiratory disease, 14, 1753466620963017. https://doi.org/10.1177/1753466620963017.

Mendes, K. D. S., Pereira, S. R. C. de C., \& Galvão, C. M. (2019). Use of the bibliographic reference manager in the selection of primary studies in integrative reviews. Texto e Contexto Enfermagem (Vol. 28). https://doi.org/10.1590/1980-265x-tce-2017-0204.

Moher, D., Liberati, A., Tetzlaff, J., Altman, D. G., \& PRISMA Group (2009). Preferred reporting items for systematic reviews and meta-analyses: the PRISMA statement. PLoS medicine, 6(7), e1000097. https://doi.org/10.1371/journal.pmed.1000097.

McGuinness, G., Zhan, C., Rosenberg, N., Azour, L., Wickstrom, M., Mason, D. M., Thomas, K. M., \& Moore, W. H. (2020). Increased Incidence of Barotrauma in Patients with COVID-19 on Invasive Mechanical Ventilation. Radiology, 297(2), E252-E262. https://doi.org/10.1148/radiol.2020202352.

Prazeres, L. E. N. dos. et al. (2021). Atuação do enfermeiro nos cuidados em Unidades de Terapia Intensiva Neonatal: Revisão integrativa da literatura. Research, Society and Development, 10(6), e1910614588. DOI: https://doi.org/10.33448/rsd - v10i6.14588.

Protti, A., Greco, M., Filippini, M., Vilardo, A. M., Langer, T., Villa, M., Frutos-Vivar, F., Santini, A., Caruso, P. F., Spano, S., Anzueto, A., Citerio, G., Bellani, G., Foti, G., Fumagalli, R., Pesenti, A., Grasselli, G., \& Cecconi, M. (2021). Barotrauma in mechanically ventilated patients with Coronavirus disease 2019: a survey of 38 hospitals in Lombardy, Italy. Minerva anestesiologica, 87(2), 193-198. https://doi.org/10.23736/S0375-9393.20.15002-8.

Rodrigues, A. N.et al. (2016). Impactos e fatores determinantes no bundle de pneumonia associada à ventilação mecânica. Revista Brasileira de Enfermagem, 69(6), 1108 - 1114. https://doi.org/10.1590/0034-7167-2016-0253.

Silva, T. G. D. et al. (2017) . Incidence of ventilator - associated pneumonia in an intensive care unit Incidência de pneumonia associada à ventilação mecânica em uma Unidade de Terapia Intensiva. Revista de Pesquisa: Cuidado é Fundamental Online, 9(4), 1121. https://doi.org/10.9789/2175-5361.2017.v 9i4.11211125 .

Souza, M. T. de, Silva, M. D. da \& Carvalho, R. de. (2010). Integrative review: what is it? How to do it? Einstein. v. 8, n. 1, 102 -106. https://doi.org/10.1590/S1679-45082010RW1134.

Sousa, L. M. M. De, Firmino, C. F., Marques-Vieira, C. M. A., Severino, S. S. P., \& Pestana, H. C. F. C. (2018). Revisões da literatura científica: tipos, métodos e aplicações em enfermagem. Revista Portuguesa de Enfermagem de Reabilitação, 1(1), 45-54. https://doi.org/10.33194/RPER.2018.V1.N1.07.4391.

Udi, J., Lang, CN, Zotzmann, V., Krueger, K., Fluegler, A., Bamberg, F., Bode, C., Duerschmied, D., Wengenmayer, T., \& Staudacher, DL (2021). Incidence of Barotrauma in Patients With COVID-19 Pneumonia During Prolonged Invasive Mechanical Ventilation - A Case-Control Study. Journal of Intensive Care Medicine, 36 (4), 477-483. https://doi.org/10.1177/0885066620954364.

Wang, T., Tang, C., Chen, R., Ruan, H., Liang, W., Guan, W., Sang, L., Tang, R., Zhong, N., \& Li, S. (2020). Clinical Features of Coronavirus Disease 2019 Patients With Mechanical Ventilation: A Nationwide Study in China. Critical care medicine, 48(9), e809-e812. https://doi.org/10.1097/CCM.0000000000004473.

World Health Organization. (2020). WHO Coronavirus Disease (COVID-19) Dashboard. https://covid19.who.int/.

Zhang, H., Zhang, Y., Wu, J., Li, Y., Zhou, X., Li, X., Chen, H., Guo, M., Chen, S., Sun, F., Mao, R., Qiu, C., Zhu, Z., Ai, J., \& Zhang, W. (2020). Risks and features of secondary infections in severe and critical ill COVID-19 patients. Emerging microbes \& infections, 9(1), 1958-1964. https://doi.org/10.1080/22221751.2020.1812437. 